\title{
An expert's opinion on what lies ahead in the field of alternative drug delivery
}

Tarun Goswami speaks to Hannah Makin, Commissioning Editor: Tarun Goswami obtained his BS (Pharmacy) in 2003 from Delhi University (India) and his PhD in Pharmaceutical Sciences in 2008 from the University of the Pacific (CA, USA). He currently works at Amneal Pharmaceuticals as a Formulation Scientist in the Transdermal Drug Delivery Group. Having published multiple abstracts and articles in the area of transdermal and oral mucosal drug delivery, his current interests include the development of drug products that are administered via alternate routes such as through the skin and oral mucosa.

First draft submitted: 6 October 2016; Accepted for publication: 21 October 2016; Published online: 16 December 2016

Keywords: sublingual microneedles $\bullet$ transdermal

How did you first become involved in the field of drug delivery?

I completed my bachelor's degree in Pharmacy from Delhi University, India, where I was introduced to many different drug delivery systems. After that I pursued a PhD in Pharmaceutical Sciences from the University of the Pacific in Stockton (CA, USA). The focus of my dissertation research was to characterize the barrier properties of the sublingual mucosa and explore it as an alternative drug delivery route. We performed various mechanistic studies using model drug molecules to understand trans-sublingual mucosa drug transport. These results were published in various peer reviewed journals. The training that I received during my $\mathrm{PhD}$ research laid the foundation for my career as a Product Development Scientist. In my industrial research and development career, I have been responsible for the product development of drugs that are either delivered via transdermal or oral mucosal routes.

What interests you about working toward finding alternative routes of delivery, such as through the skin \& oral mucosa?

The majority of drugs are delivered either by the oral or parenteral route. Many drugs are not amenable for oral delivery as they are subject to high first-pass metabolism or gastric degradation. The alternative routes of drug delivery, such as transdermal and the oral mucosa, avoid these problems and provide added benefits, such as near zero order release profiles with transdermal route or quick onset of action with sublingual route. Moreover, transdermal route presents a viable, noninvasive route wherein drug absorption can be maintained for multiple days at a time. A lot of basic academic research has been conducted in the area of alternative routes of drug delivery, but this academic research has not translated into a lot of commercialized products. Currently, there are a dozen or so products in the market in which drugs are delivered using these alternative routes. There are a lot of constraints (such as requirement for specific physicochemical properties of the drug and complex manufacturing) associated with these alternative routes,

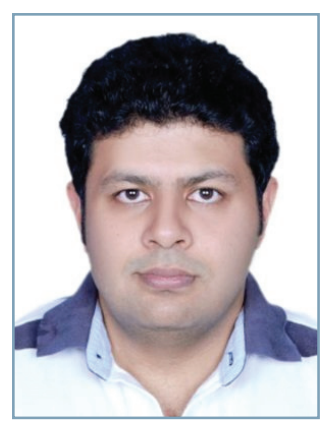

Tarun Goswami Amneal Pharmaceuticals Transdermal drug delivery group 1 New England Ave Piscataway, NJ 08854 USA Tarungos@gmail.com 
which makes $R \& D$ in these areas both challenging and interesting.

\section{Q Can you update us on the current work you} \& your research group are undertaking?

Currently our research group is involved in transder$\mathrm{mal}$ and buccal/sublingual product development. We are developing products that will be submitted via ANDA (Abbreviated New Drug Application) or 505 B-2 pathways to the regulatory agencies for final US FDA approval. Our objective is to develop improved transdermal products with elegant design, better adhesion and lower residual drug load. Specifically, we are trying to reduce the drug load in patches that employ potent drugs, such as opioids. Less residual drug load after use of these patches will prevent misuse and help our environment due to minimal drug contamination in our waste streams.

\section{With regards to your recent research on} sublingual delivery systems, why do you think so few drugs have been found to be successfully delivered through this method of delivery?

Not all drugs have the desired physicochemical properties required for delivery across the sublingual mucosa. Generally speaking, small highly lipophilic molecules administered at a dose of $<50 \mathrm{mg}$ are most suitable for this route of drug delivery. From a product development standpoint, the sublingual mucosa is not the preferred route of delivery during the early stage of clinical development of a drug. Due to money and time issues, drug companies prefer the conventional, less risky, oral or parenteral routes. Selection of the sublingual route is more of an afterthought when there are specific problems associated with the drug molecule; such as a high first-pass metabolism; or perhaps a clinical need, such as a requirement for immediate action (e.g., with nitrates in the case of angina pain). In addition, the sublingual region is continually washed by saliva as there is constant movement of the tongue, therefore, it is not suitable for the prolonged retention of drug products.

\section{Q Why do you think the field of transdermal drug delivery systems has grown so much in recent decades?}

People have been administering medicinal substances via the skin for as long as we can remember. Initially, drugs were administered on skin for local action use only. With advances in technology and growing evidence of systemic absorption via the transdermal route, transdermal patches started becoming popular. Clinicians and patients like these small, inexpensive, painless and aesthetic delivery systems, which provide a constant blood level profile over multiple days.
In a clinical setting, these features especially come in handy for unconscious or uncooperative patients who do not want to ingest pills or comply with the suggested therapy. With further developments in the field, physical enhancement means, such as iontophoresis, sonophoresis, heat and now microneedles (MNs) are being used to increase the number of drugs that can be delivered via the transdermal route. These constant advancements have kept the interest in this area alive.

You recently wrote a review discussing pressure-sensitive adhesives [1]. How are issues in finding the ideal pressure-sensitive adhesive critical to the field of transdermal drug delivery system development?

Pressure-sensitive adhesives are the most critical excipients in transdermal delivery systems. They are multifunctional excipients responsible not only for adhesion, but also drug release and stability of the transdermal delivery system while maintaining its viscoelastic character. These adhesives provide varying degrees of cross linking, glass transition temperatures, functional groups and resin-to-polymer ratios, which can potentially influence the final drug formulation in terms of its safety (irritation and sensitization) and performance (physical properties and drug release). Either a single or mixture of adhesives can be used to optimize the prototype transdermal delivery system. Adhesive properties such as adhesiveness, cohesiveness, tack, viscosity, molecular weight (MW), extent of cross-linking and solubility can affect the performance of the patch. In addition, the residual solvent content, residual monomer content and impurities can also affect both the safety and performance of the patch. Therefore, the effect of key adhesive properties on the safety and performance of the patch warrants comprehensive experimentation and understanding during the formulation development process.

\section{What are the current major issues that} researchers are facing in your field of work?

There have been numerous reported problems with respect to transdermal product quality and performance. There have been delays in drug approval and numerous recalls over the years. From a research and developmental perspective, the recalls have been related to issues such as dose dumping from leaky fentanyl reservoir patches, crystallization in rotigotine patches and release liner removal issues with methylphenidate patches. These issues have resulted in increased regulatory scrutiny and a need for 'quality by design'-based approach in product development.

Using passive delivery approaches, only a few drugs are viable candidates for transdermal delivery. Large 
hydrophilic molecules with MWs $>500$ Da cannot be delivered across the skin. In addition, generally speaking, delivering $>10 \mathrm{mg}$ of drug daily via the passive transdermal route still remains a challenge.

\section{Do you have any suggestions as to how these challenges should be overcome?}

The need of the hour is for regulatory agencies, industry and academia to harmonize and come up with a clear guideline for the development and approval of these novel transdermal systems such as MNs. From a development perspective, companies have to implement quality by design strategies early on in the development process to avoid surprises later on. Mistakes have been made in the past but industry as a whole has to learn from these mistakes and make sure that the same mistakes are not repeated. Also, a lot of effort is being put into developing active transdermal approaches such as $\mathrm{MN}$ based approaches for the delivery of macromolecules through the skin. There are numerous clinical trials in progress but so far no $\mathrm{MN}$ based product has been commercialized.

A very limited number of drugs have the desired physicochemical properties required for transdermal route absorption. Recently, however, the drug rotigotine was developed specifically for transdermal delivery. The passive transdermal area needs more drugs such as rotigotine to provide efficient delivery systems.

\section{You mentioned in recent research that a major} challenge in developing drug delivery systems is a lack of mutual understanding between regulatory bodies \& those working in industry [2]. In detail, how does this problem challenge current research \& how do you think it can be overcome?

Most drug research these days looks at macromolecules such as proteins, peptides and other biological drugs. These molecules cannot be delivered via the conventional passive transdermal route due to their size and lipophilicity. To overcome the permeability barrier, physical means, such as MNs are being used to perturb the skin layers, which would facilitate the delivery of these macromolecules. There are a lot of clinical trials going on using these physical enhancement approaches, such as MNs. Still, a lot of progress is required in the area of scaling up these products to get regulatory approval. Due to the novel nature of this technology, expectations of regulatory agencies regarding approval of these new delivery systems are not clear. There are impending regulatory issues that need guidance and clarity, such as sterility/low bioburden requirements, content uniformity, dose reproducibility, immunological reactions, potential of re-use, need of an applicator and long-term safety profiles. There is a need for mutual understanding and concurrence between regulatory agencies and industry to come up with a development pathway for these products. I think the first set of approved MN products would set the tone for critical quality testing and regulatory requirements for subsequent products.

\section{If researchers overcame these issues, what do} you think lies in the future for transdermal \& oral mucosal delivery systems?

In the future, most of the new drugs will be biological/ protein based. These drugs cannot be delivered via the oral route and therefore the parenteral route is the preferred choice. There is always a need and interest in the medical community to find noninvasive means of delivering these macromolecules. Approval of MN-based products will open the door to a wide variety of macromolecules and even difficult-to-deliver small molecules across the skin.

Oral mucosal delivery will be a viable alternative for drugs that require specific clinical need, such as when the immediate delivery of drugs into the bloodstream and avoidance of first-pass metabolism is Advances in formulation designs, such as fast-dissolving films and mucoadhesive delivery systems, will result in oral mucosa delivery being a viable alternative route for some drugs and a tool for the life-cycle management for existing molecules. Currently, BioDelivery Sciences International has used its BEMA ${ }^{\circledR}$ (BioErodible Mucoadhesive) technology to develop a range of buccal transmucosal films such as Bunavail ${ }^{\mathrm{TM}}$ (BioDelivery Sciences International, Inc.Raleigh, NC, USA) . In addition, drugs are also being formulated as sprays to provide effective oral-mucosal delivery, for example, sublingual fentanyl spray by Insys therapeutics (Insys TherapeuticsPhoenix, AZ, USA).

\section{What advice would you give a young} scientist who is just beginning a career in transdermal/buccal drug delivery research? My advice for young scientists is to keep yourself abreast of the latest happenings in your field of research. Skin as such is a protective organ and not amenable for the delivery of drugs. This makes the successful delivery of drugs via skin a challenging affair. Transdermal delivery is a very niche area and sometimes the development and approval pathways become long and tedious. In my view, the key is to show perseverance and stick with the product till the end, as there is no bigger satisfaction for a researcher to see his or her idea get from bench to bedside.

\section{Disclaimer}

The opinions expressed in this interview are those of the interviewee and do not necessarily reflect the views of Future Science Ltd 
Financial \& competing interests disclosure

T Goswami has no relevant affiliations or financial involvement with any organization or entity with a financial interest in or financial conflict with the subject matter or materials discussed in the manuscript. This includes employment,

\section{References}

1 Lobo S, Sachdeva S, Goswami T. Role of pressure-sensitive adhesives in transdermal drug delivery systems. Ther. Deliv. 7, 33-48 (2016). consultancies, honoraria, stock ownership or options, expert testimony, grants or patents received or pending, or royalties.

No writing assistance was utilized in the production of this manuscript.

2 Sachdeva S, Lobo S, Goswami T. What is the future of noninvasive routes for protein- and peptide-based drugs? Ther. Deliv. 7, 355-357 (2016). 